\title{
Collecte, gestion et valorisation des données sur les formations et les gîtes à silex en France : pour des plateformes cartographiques et de publications partagées.
}

\section{Collecting, managing and promoting data on flint deposits and sites in France: for shared cartographic and publishing platforms.}

\author{
Christophe Tuffery ${ }^{1}$, Paul Fernandes ${ }^{2-3}$, Vincent Delvigne ${ }^{4-3}$, Céline Bressy-Leandri ${ }^{5}$, \\ Stéphane Renault ${ }^{6}$
}

\footnotetext{
${ }^{1}$ Inrap, Direction Scientifique et Technique, 121 rue d'Alésia, CS 20007, 75685 Paris Cedex 14, France

${ }^{2}$ SARL Paléotime, 6173 rue Jean Séraphin Achard Picard, 38350 Villard-de-Lans, France

${ }^{3}$ UMR 5199 - PACEA, Université de Bordeaux, bâtiment B18, Allée Geoffroy Saint-Hilaire, 33615 Pessac Cedex, France

${ }^{4}$ Service de Préhistoire, Université de Liège, place du XX août, 4000 Liège, Belgique

${ }^{5}$ Service régional de l'archéologie, DRAC de Corse, Villa San Lazaro, 1 chemin de la Pietrina, CS 10003, 20704, Ajaccio

Cedex 9, France ; TRACES, UMR 5608

${ }^{6}$ Laboratoire Méditerranéen de Préhistoire Europe-Afrique, UMR 7269 Aix-Marseille Université - CNRS - MCC - Inrap, MMSH - 5, rue du Château de l'Horloge - BP 647 - 13094 Aix-en-Provence Cedex 2 France
}

\begin{abstract}
RÉSUMÉ. Depuis une dizaine d'années, plusieurs Projets Collectifs de Recherche (PCR), résolument interdisciplinaires, ont produit un ensemble de données descriptives des principaux silex associés à leurs gîtes primaires et secondaires dans le sud et le centre de la France selon une nouvelle méthode de caractérisation des matériaux siliceux. Ces travaux sont en cours de convergence et d'harmonisation dans le cadre d'un Groupement de Recherche (GDR) soutenu par le CNRS et le Ministère de la Culture pour cinq années. Ce nouveau cadre permet à la communauté des scientifiques concernés dans divers domaines d'envisager une valorisation des données collectées depuis plusieurs années sur les géoressources lithiques, en adoptant des pratiques harmonisées aux différentes étapes du cycle de vie des données, depuis leur collecte jusqu'à leur publication en ligne, dans le respect des principes $F A I R^{1}$.

ABSTRACT. Over the past ten years, several resolutely interdisciplinary Projets Collectifs de Recherche (PCR), have produced a set of descriptive data on the main flints associated with their primary and secondary deposits in southern and central France using a new method for the characterization of siliceous materials. This work is in the process of convergence and harmonization within the framework of a Research Group (GDR) supported by the CNRS and the Ministry of Cultural Heritage for five years. This new framework allows the community of scientists concerned in various fields to consider enhancing the value of data collected over several years on lithic georesources by adopting harmonized practices at different stages of the data life cycle, from collection to online publication, in accordance with FAIR principles. MOTS-CLÉS. Webmapping, matériaux siliceux, géoressources, bases de données.

KEYWORDS. Webmapping, siliceous materials, georesources, databases.
\end{abstract}

La caractérisation pétrographique des matériaux siliceux utilisés par les populations préhistoriques est aussi ancienne que l'archéologie préhistorique elle-même. Aujourd'hui, l'étude des provenances des géoressources s'inscrit dans un ensemble d'analyses pluridisciplinaires portant sur les paléoenvironnements, les itinéraires de déplacement des populations, les choix dans les matériaux ou encore les conditions d'extraction, d'utilisation et d'abandon des matériaux lithiques par les populations préhistoriques.

${ }^{1}$ Findable, Accessible, Interoperable, Reusable traduit par Facilement trouvable, Accessible, Interopérable et Réutilisable 
D'importantes avancées ont été rendues possibles au cours des dernières années dans la caractérisation des matériaux siliceux et l'identification de marqueurs physico-chimiques des différentes étapes de leur mise en place et de leur évolution dans le temps et dans l'espace, dans les gîtes primaires et dans les gîtes secondaires (Fernandes P., 2012). À partir de ces travaux, une méthode harmonisée a été mise au point et a été réutilisée dans plusieurs PCR « Réseaux de lithothèques » pour les régions Auvergne-Rhône-Alpes, Nouvelle Aquitaine, Centre Val-de-Loire et prochainement Ile-deFrance.

Après un important travail entre spécialistes des matériaux siliceux, il a été possible de mettre en place une fiche de terrain rassemblant les données descriptives minimales (52 champs; Delvigne et $a l$. sous presse) pour la description de tous les gîtes à silex. Cette fiche d'observation permet d'alimenter une base de données, développée initialement sous Access et qui devrait être migrée prochainement sur une solution open source, qui comporte diverses tables (gîtologie, taphonomie, pétrographie) permettant d'établir une partie de la carte d'identité de chaque type de matériau siliceux.

En parallèle, une méthode harmonisée pour la numérisation des contours cartographiques des formations à silicites ${ }^{2}$ a été mise au point et utilisée dans plusieurs régions. Elle est basée sur l'analyse des données acquises (observations, échantillonnages) sur le terrain. Les couches géoréférencées ont été numérisées avec ArcGIS et QGis à partir de l'affichage au 1/10 000 et d'une lecture critique des cartes géologiques au 1/50 000 disponibles sur le service web cartographique (WebMapService ou WMS) Infoterre du Bureau de Recherches Géologiques et Minières (BRGM). La numérisation est systématiquement validée par un expert, souvent un géologue, comme cela a été le cas pour la Dordogne (J-P. Platel, ancien géologue du BRGM) et peut nécessiter des retours sur le terrain pour vérifier certains contours ou lever des doutes d'attribution géologique.

\section{Les données et les outils existants}

La fiche de terrain a été mise au point au format .xls dans un tableur Excel (elle est également disponible au format .ods pour LibreOffice) et peut être renseignée comme un formulaire (fig.1).

\footnotetext{
${ }^{2}$ Le terme de silicite, introduit par A. Prichystal (2010) en Europe centrale et orientale, désigne l'ensemble des roches ayant subi une silicification d'origine chimique, biochimique ou diagénétique. Il permet de s'affranchir du terme silex, dont le sens (roches hypersiliceuses développées dans les formations marines, lacustres et palustres, résultant en grande partie d'un remplacement des carbonates et des bioclastes, constituées de grains fins, à cassure lustrée, dissociées du sédiment d'origine et contenant au moins $90 \%$ de silice, le plus souvent de la calcédoine) est sujet à controverse et à des incompréhensions tantôt qu'il est employé par les géologues, les pétrographes, les sédimentologues ou les archéologues.
} 


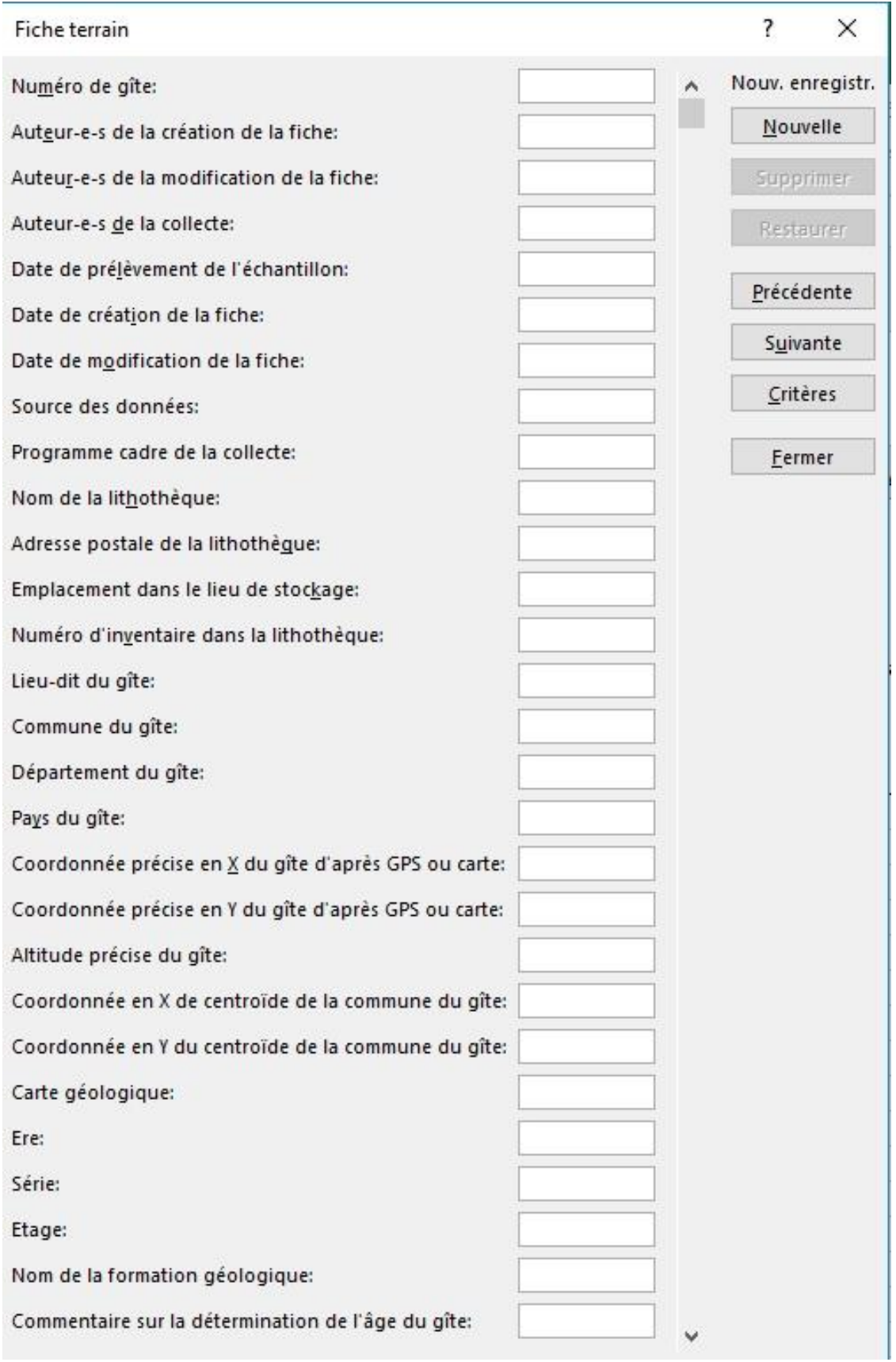

Figure 1. Extrait du formulaire de saisie des données sur les gîtes à silicites dans un tableur

Une autre version a été développée à l'aide de l'application 123Survey for ArcGIS d'ESRI qui permet de saisir des données sur le terrain avec un dispositif de type smartphone ou tablette sous Android ou iOS (fig.2). Les données peuvent être directement enregistrées et partagées depuis le terrain s'il existe une connexion par l'internet mobile ou elles peuvent être partagées et synchronisées ultérieurement si la connexion à l'internet mobile sur le terrain n'est pas disponible. 
Date de création de la fiche*

Date de modification de la fiche*

dd.mm.yyyy

Source des données*

Programme cadre de la collecte*

-Sélectionnez-

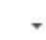

Nom de la lithothèque*

Adresse postale de la lithothèque*

Figure 2. Extrait du formulaire de saisie sur le terrain de données sur les gîtes à silicites à l'aide de l'application 123Survey for ArcGIS

À ce jour, les formations à silex du département de la Dordogne ont été entièrement numérisées grâce à un soutien du Centre National de la Préhistoire à Périgueux. Celles sur la région Centre-Val-deLoire l'ont été partiellement et devraient être prochainement complétées. D'autres zones ont commencé à être couvertes en utilisant toujours le même protocole pour la numérisation des contours des formations à silex (région PACA, ARA, etc.).

Les données sur les formations ont été partagées au sein du collectif des chercheurs sur une application de cartographie en ligne s'appuyant sur la plateforme ArcGisOnLine (AGOL) et les outils de construction d'application Web (Web AppBuilder for ArcGIS). Cette application propose l'affichage de la couche des formations à silicites et de la légende associée ainsi que celui de plusieurs autres couches de données: gîtes à partir de la fiche terrain mentionnée précédemment, services web cartographiques comme celui du BRGM, etc. (fig.3). 


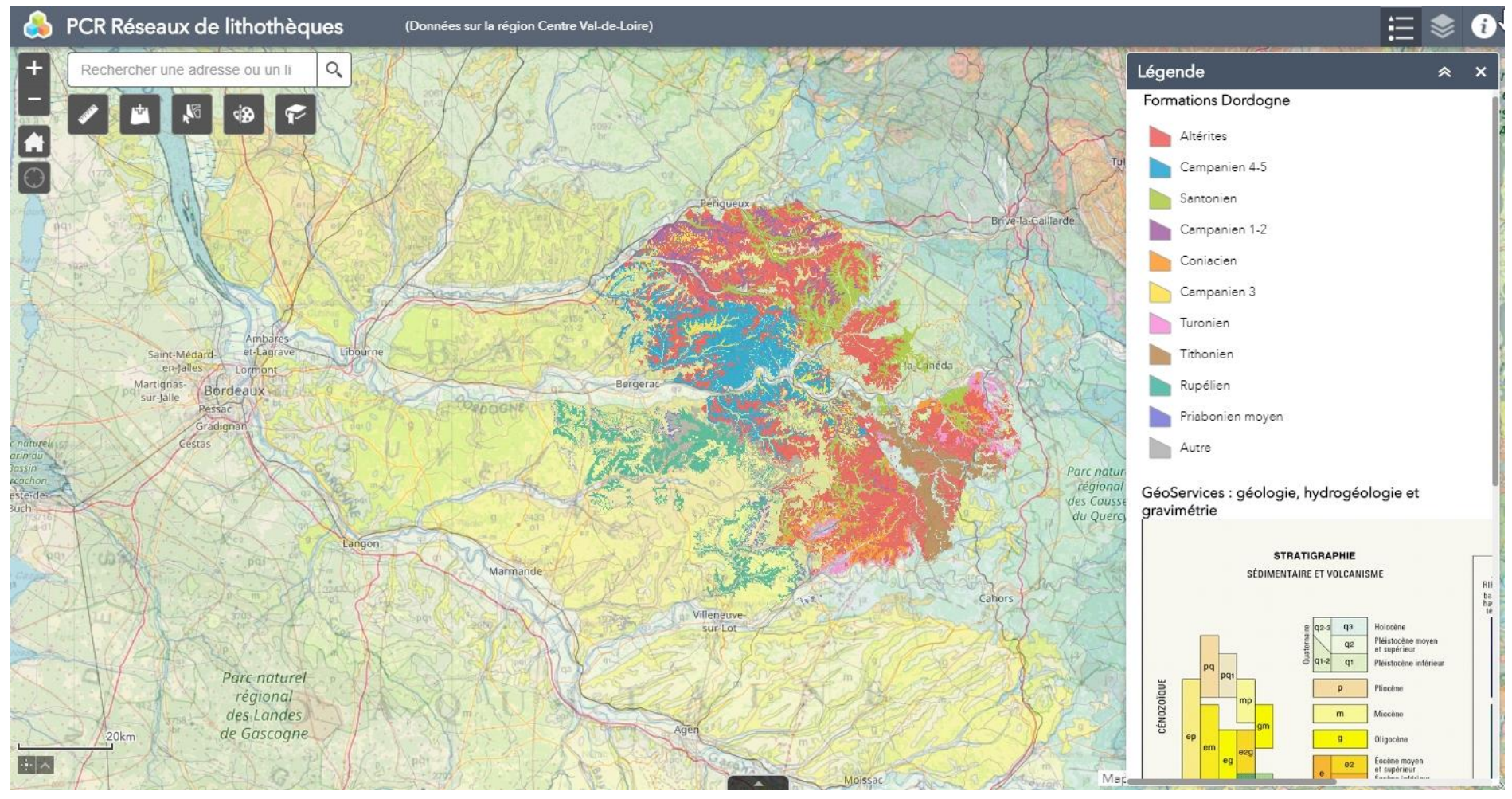

Figure 3. Formations à silicites numérisées sur l'ensemble du département de la Dordogne et affichées en complément du service web cartographique des cartes géologiques (source : Infoterre du BRGM

Aussitôt saisies sur le terrain à l'aide de l'application 123Survey for ArcGIS, les données peuvent être affichées et partagées dans l'application cartographique (fig. 4).

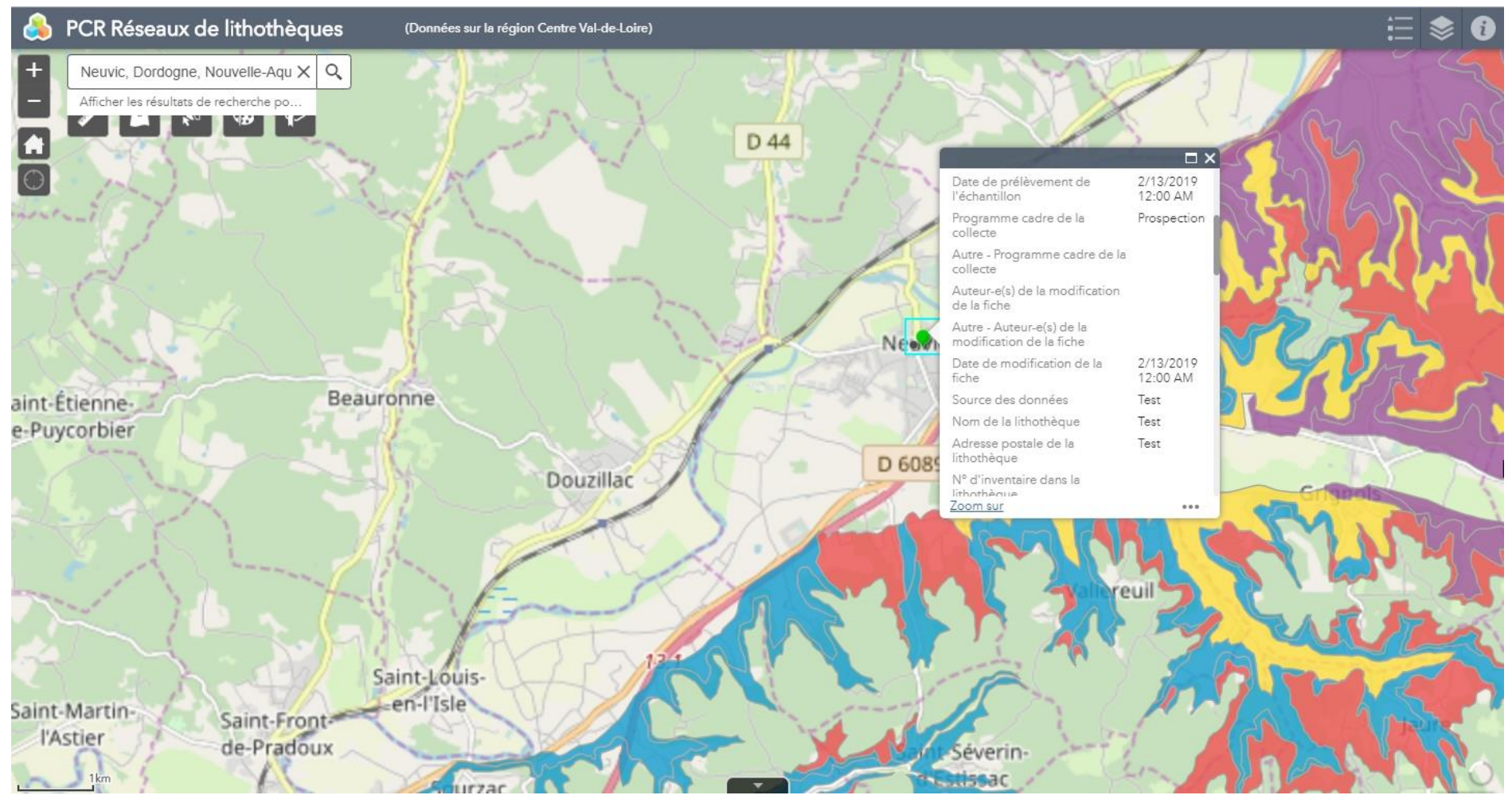

Figure 4. Consultation des données associées à un gîte et affichage des formations à silicites dans la région de Neuvic-sur-l'lsle (Dordogne) 


\section{Vers une valorisation et un partage en ligne des données}

Une fois constituées, les données sur les formations et sur les gîtes à silicites peuvent être consultées et, si besoin, corrigées et complétées sur smartphones et tablettes avec un simple navigateur Internet ou avec l'une des applications proposées par ESRI et disponibles sous Android (depuis Play Store) ou iOS (depuis Apple Store) :

- Explorer for ArcGIS (fig. 5)

- Collector for ArcGIS

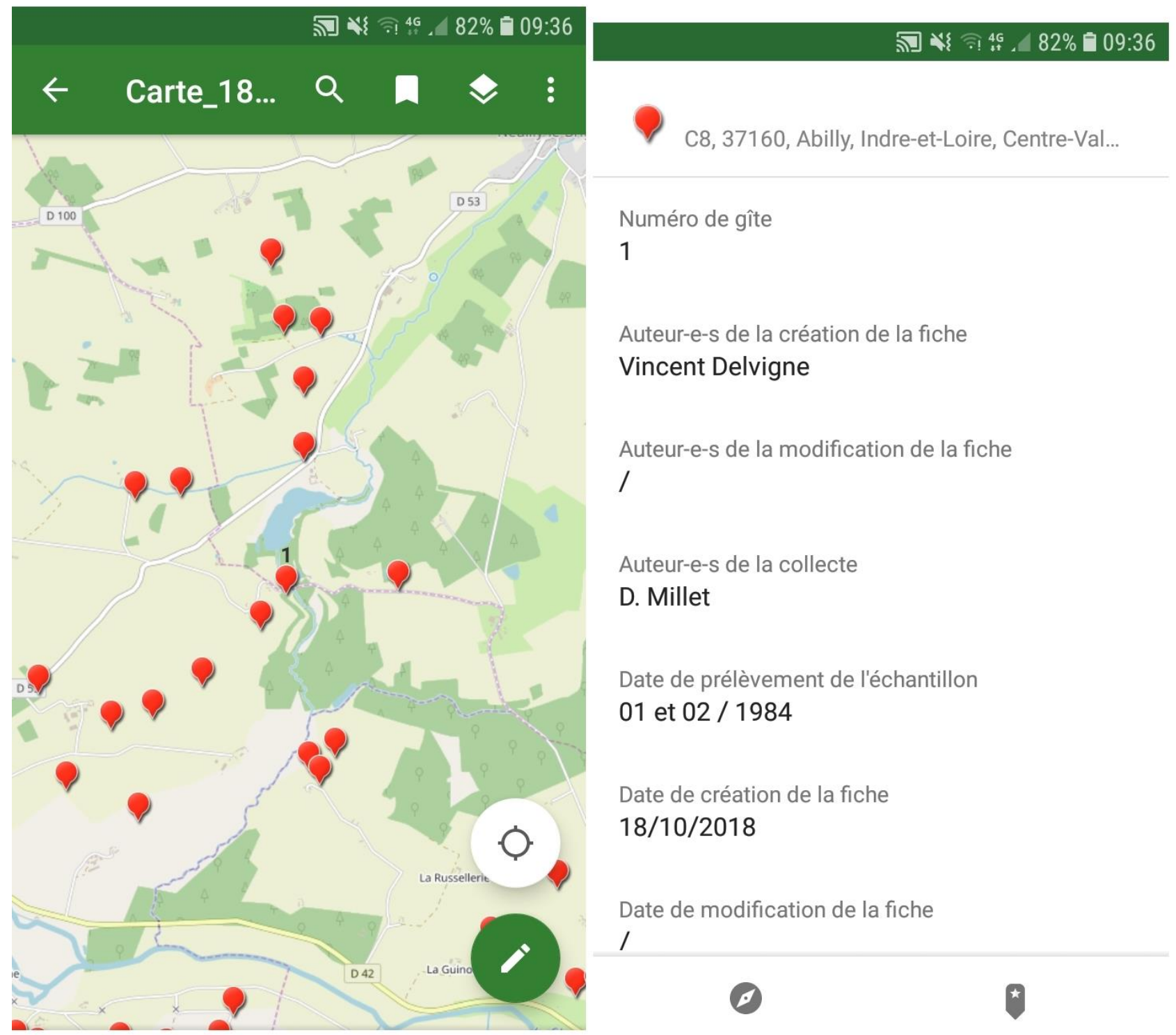

Figure 5. Affichage dans Explorer for ArcGIS sur tablette ou smartphone des données descriptives associées à un gîte.

Ces applications sont très simples à utiliser et peuvent être personnalisées autant que de besoin sans avoir à écrire la moindre ligne de code informatique. Outre le volet cartographique, ces applications permettent de consulter, de modifier, de compléter les données descriptives et d'accéder, par des liens de type URL, à des ressources documentaires multiples, en accès libre ou partagé comme des photos d'échantillons de silex prélevés sur le terrain, des photos de gîtes (fig. 6 ) ou de nombreuses autres ressources documentaires dans divers formats. 


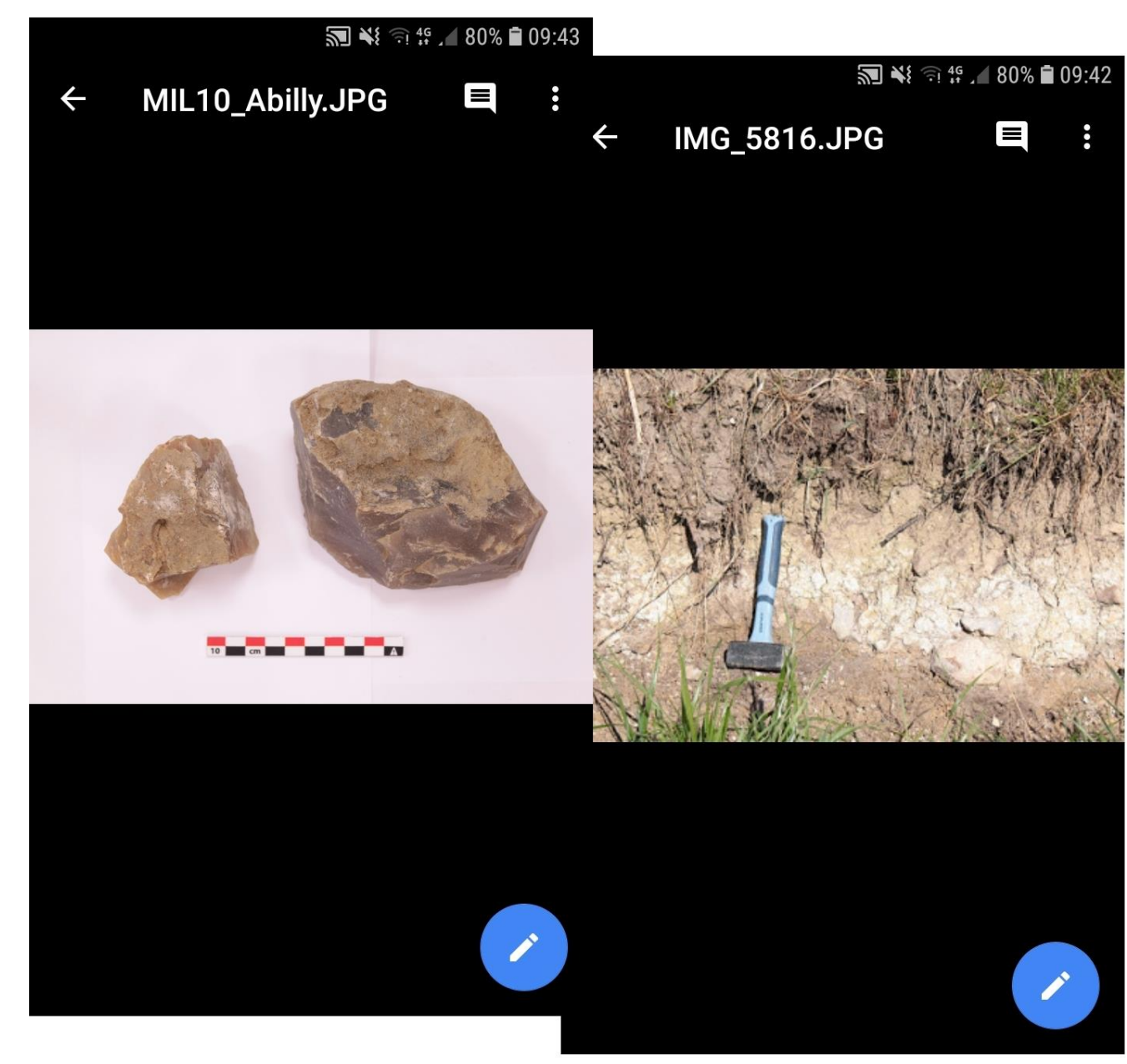

Figure 6. Affichage de photos d'échantillons de silex et de gîte dans l'application Explorer for ArcGIS sur tablette ou smartphone

\section{Perspectives : le partage et la diffusion des données et des métadonnées}

La dimension spatiale des différentes données sur les silicites, regroupées par les divers PCR «Réseaux de lithothèques » puis dans le cadre du nouveau GDR «Silex » ${ }^{3}$, permet d'ores et déjà d'enrichir les raisonnements sur les rapports entre la localisation des géoressources siliceuses et les paléoenvironnements au cours de la Préhistoire (Delvigne V., 2019, Fernandes P., 2019, Morala A., 2019). Des hypothèses sur l'itinéraire parcouru par le silex avant sa collecte par l'homme préhistorique peuvent être émises, alimentant des études paléogéographiques renouvelées, en particulier sur l'organisation des sociétés préhistoriques, leur gestion de l'espace et leur rapport aux territoires.

Les travaux qui seront conduits dans le cadre du nouveau GDR «Silex » vont pouvoir profiter pleinement des divers protocoles de numérisation de données cartographiques et de saisie de données descriptives déjà éprouvés. Ces travaux vont également pouvoir tirer parti rapidement des divers outils de saisie de données et des diverses applications cartographiques développées pour un usage en ligne et en situation de mobilité. Ces divers outils seront étendus soit sur la base des mêmes choix technologiques que ceux déjà mis en œuvre, soit en faisant le choix généralisé de solutions open source. Chacun des jeux de données et de métadonnées pourra bénéficier d'une diffusion contrôlée par la publication de data papers, aboutissement des différentes étapes d'une chaine intégrée de publication. La mise en place de cette chaîne, ainsi que l'élaboration d'un modèle de data paper assurant la qualification, les conditions de réutilisation et la traçabilité des jeux de données primaires, pourront s'inscrire au sein de la plateforme ARCADIIS (Archaeological Data Infrastructure for Interoperability and Sharing), en cours de déploiement (Renault et Nouvel 2018). Conçue comme un

\footnotetext{
${ }^{3}$ GDR «Silex » ${ }^{\circ} 2059$ accepté pour 5 ans, soutenu par le CNRS (INEE, section 31) et le Ministère de la Culture, placé sous la coordination de Céline Bressy-Leandri
} 
$h u b$ éditorial adoptant les standards du web sémantique et répondant aux principes FAIR, ArcaDIIS accompagne la structuration et la qualification des jeux de données en préalable à leur dépôt, pour en assure leur paternité. L'exploitation de vocabulaires contrôlés (thésaurus PACTOLS) et normalisés (SKOS) assurent une indexation et une annotation automatique à travers un module de gestion de terminologies. Les principes qui régissent la plateforme s'inscrivent pleinement dans le mouvement de la science ouverte. Elle entend en outre accompagner les projets/programmes de recherche tout au long de son cycle de vie pour permettre l'évaluation de toutes productions qui y sont liées.

\section{Références bibliographiques}

Delvigne, V., Fernandes, P., Tuffery, C., Angevin, R., Lethrosne, H., Aubry, T., Creusillet, M.- F., Depont, J., Le Bourdonnec, F.X., Lafarge, A., Liabeuf, R., Mangado-Llach, X., Moncel, M.- H., Philippe, M., Piboule, M., Primault, J., Raynal, J.-P., Recq, C., Sanchez de la Torre, M., Teurquety, G., Verjux, C. (sous presse) - Grand-Pressigny was not alone: acquiring and sharing data about raw materials in the collective research project "Réseau de lithothèques en région Centre-Val de Loire" (France). Journal of lithic studies.

Delvigne V. (dir.), 2019, Projet collectif de recherche "Réseau de lithothèque en Centre Val-de-Loire". Rapport d'activité 2018,182 pages

Fernandes P., 2012, Itinéraires et transformations du silex : une pétroarchéologie refondée, application au Paléolithique moyen. Thèse de doctorat en Préhistoire soutenue le 19/06/2012 à l'Université de Bordeaux 1. 2 vol. 623 pages

Fernandes P. (dir.), 2019, Projet collectif de recherche "Réseau de lithothèque en Auvergne - Rhône-Alpes ". Rapport d'activité 2018, 200 pages

Morala A. (dir.), 2019, Projet collectif de recherche "Réseau de lithothèque en Nouvelle Aquitaine". Rapport d'activité 2018, 114 pages

Prichystal A., 2010, Classification of lithic raw materials used for prehistoric chipped artefacts in general and siliceous sediments (silicites) in particular: the Czech proposal, Archeometriai Muhely, 2010 (3), p. 177-182.

Renault S. et Nouvel B. 2018, Renault Stéphane, Nouvel Blandine 2018, ArcaDIIS (Archaeological Data Infrastructure for Interoperability and Sharing). Rendre visibles et évaluables les données de l'archéologie, in Ouvrir la Science! Premières journées Nationales de la Science couverte. De la stratégie à l'action - 4-6 décembre 2018, Paris (Poster) https://jnso2018.sciencesconf.org/data/pages/ArcaDIIS.pdf 